\title{
A Study of the Entropy Production in Physical Processes from a New Perspective of the Energy Structure
}

\author{
Saeed Shahsavari ${ }^{*}$, Mehran Moradi² \\ ${ }^{1,2}$ Department of Mechanical Engineering, Isfahan University of Technology \\ Isfahan, Iran \\ *Corresponding author's email: s.shahsavari [AT] me.iut.ac.ir
}

\begin{abstract}
When a physical process is performed, identifying the generated entropy can be used to investigate the irreversibility. But for this mean, from the perspective of the Boltzmann equation, both all microstates and macrostates must be studied. In fact, it is needed that all particles energy level to be investigated. Therefore, to investigate entropy in configurationally systems using the Boltzmann equation, a very large volume of calculations is required. In this study, we try to extract a way to investigate entropy production without the need to study all particles (or sub-structures). For this purpose, at first, a macroscopic energy structure equation "as an equation that shows the energycomponents of the system activated in the performed process as well as their dependence" is presented. As a study on the irreversibility (or entropy production) in physical systems, its structure and components are studied. Writing equations in the energy space of the system makes it possible to study the structure of irreversibility. Then using a new macroscopic quasi-statistical approach, the irreversibility and its structure in physical processes are investigated. Macro energy components of the system are used for this investigation and energy structure is studied base on them. Finally, a new macroscopic definition of the generated entropy is extracted using a new energy structure equation as well as dependent and independent macroscopic energy component concepts. Also, why and what entropy can be generated, from the perspective of the presented macroscopic energy structure equation are studied. In fact, this paper investigates the generated entropy structure in physical systems using macroscopic system energy components and takes a new approach to why and what irreversibility is occurred during the physical process. Therefore, presented equations can be used for investigating the irreversibility in configurationally physical systems without the need to study all its sub structures. Also, from the extracted equations, it can be concluded that entropy is generated because of the existence of the dependent energy components in the energy structure equation of the system, and this generated entropy depends on the variation of these components as well as the amount of the applied energy to the system and its conditions. Due to the kinematic theory of dissipated energy, these results are in the same line with the different formulations of the second law of thermodynamics.
\end{abstract}

Keywords--- Thermodynamics; Configurationally physical systems; Entropy production; Irreversibility components; Energy Structure; Energy components; Dependent components

\section{INTRODUCTION}

Principles of conservation examine the quantitative concepts in physics. Such as energy conservation, mass conservation, linear and angular momentumconservation, etc., which can be applied to physical phenomena, and every physical process must be performed in such a way that these principles are satisfied. The only physical law that considers the concept of direction of processes is the second law of thermodynamics [1]. Before the first law was introduced, Carnot introduced reversible processes in him paper, and accordingly could be considered the founder of this law [2].

Subsequently, the second law of thermodynamics gained a very important place in science, so that it undoubtedly became the most important physical law. As Einstein stated, the only law that will never collapse is the second law of classical thermodynamics $[1,3]$. This law has a special place in all branches of science as a constitution.

The second law defines the physical quantity as entropy. According to this law, the entropy of an isolated system is constantly increasing, and in fact, increasing entropy means the passage of time. From a classical thermal theory point of view, the second law is interpreted in such a way that the amount of available work is always decreasing, and instead the amount of thermal energy increases [1]. However, in the attitude of material structures, increasing entropy increases irregularity [4]. Also from the statistical physics point of view, increasing entropy means increasing the number of available states [5], and froma statistics quantumpoint of view, is interpreted as an increase in the number of energy levels [6]. 
Two main formulations that have been extracted to the second law from the classical thermodynamics point of view are Kelvin-Plank and Clausius formulations [7,8,9]. Kelvin-Plank and Clausius formulations are as sertions about impossible processes, while some formulations to the second law are presented which rely on as sertions about the possible processes [9]. Als o, Caratheodory formulation is one of the other formulations to the second law from the clas sical thermodynamics point of view [10-13]. Clausius uses entropy quantity to formulate the second law [14]. Based on his perspective, in all performed physical processes, entropy must be increased. Therefore, generated entropy can be known as a physical direction for physical processes $[15,16]$. In the Clausius perspective, the physical concept of entropy is the loss of useful energy and exchange it to the thermalenergy [9,17-19].

In classical thermodynamics, the entropy formulation is used to equilibrium states and no attempt is made to define it otherwise [9]. Although in the statis tical physics point of view, entropy is formulated as a physical quantity that depends on the available probability of the physical system at a s pecial particles energy level and can be used to equilibriumas well as non-equilibrium states. Also, the absolute value of entropy can be calculated by investigating the microstates of the system [20,21]. Therefore, due to the statistical physics point of view, it can be concluded that the second law will be equivalent to the fact which the probability of the system will be increased always [20].

Also, there are generalized formulations to the second law [21-24]. In thesecases, it is needed that all sub-structures of the system to be investigated. In fact, each sub-structure must be studied to calculate the entropy of the system.

In this study, a new macroscopic quasi-statistical perspective on why and what entropy is generated during physical processes is presented. Using the energy components of the system an energy structure equation is presented and based on it, without the need to investigate all sub-structures of the system, the generated entropy structure, and its macroscopic components are investigated.

\section{ENTROPY IN CLASSICAL THERMODYNAMICS AND STATISTICAL PHYSICS POINT OF VIEW}

The clas sical thermodynamics examines the second law from point of view of thermal energy on a macroscopic scale and states that in physical processes, some of the available work is always converted into thermal energy. Therefore the second law can be expressed as follows [4]:

$\oint \frac{\delta Q}{T} \leq 0$

Where $\delta Q$ is the heat exchanged at temperature $T$. If the system has an almost continuous dis tribution of matter, so that quantities such as density can be attributed to it, then the second law can be written using the concept of entropy as follows:

$\frac{d}{d t} \int_{p} \rho s d V \geq \int_{p} \rho e d V-\int_{\partial p} \frac{q_{i} n_{i}}{T} d a$

Where $p$ is the material particle of the systemand als o $\partial p$ is the surface containing the material (particle) of the $p$. Also,e as the entropy entry rate into the system by external sources per unit mas $s, q_{i}$ is the heat flux vector passing through the unit surface, $\rho$ is density, $n_{i}$ is the surface normal vector, $T$ is temperature and $s$ is the entropy. Also, the equation(2) can be rewritten as follows in its differential form:

$\frac{d s}{d t}-e-\frac{1}{\rho}\left(\frac{q_{i}}{T}\right)_{, i} \geq 0$

Equality mode is for reversible processes and non-equality mode represents irreversible processes. To examining the irrevers ibility from the clas sical thermodynamic point of view, the following equation can be considered as one of the most important equations [25]:

$\overrightarrow{I_{k}}=\sum_{l} L_{k l} \vec{F}_{l}$

Where $\overrightarrow{I_{k}}$ is a set of general currents and $\vec{F}_{l}$ is thermodynamic forces. $L_{k l}$ is also a matrix of kinematic coefficients. . Also, the $L_{k l}$ matrix is required to satisfy the symmetry equations in the OSAGER equations [26]. In this point of view, the irreversibility is examined using investigating the sub-structures.

However, in reversible processes where there is no entropy generation, entropy is written as follows according to the heat exchanged as well as the systemtemperature:

$T d s=d Q$

Classical thermodynamics is incapable of calculating the absolute value of entropy and examines its relative changes. According to the second law, $d s \geq \frac{d Q}{T}$ or $d s_{g e n} \geq 0$, which $s_{g e n}$ is the entropy generation, is always positive. The entropy 
generated is a measure of the amount of available work (or the amount of irreversibility) lost. As a result, some of the available work is converted into thermal energy, and in fact into other components of energy. The amount of irreversibility is considered as $T_{0} \delta S_{g e n}$ that is defined based on entropy generation and theenvironment temperature [4].

Statistical mechanics investigates the second law and entropy from a microscopic point of view. Given Boltzmann's perspective, it is neces sary to consider the entropy of a systemas a function of the probability that the systemwill reach that particular state at the desired energy level [27]:

$S=f(W)$

Where $S$ is entropy and $W$ is the probability in the desired state.

If we combine different states, then it is necessary to add their entropy, although the probability will be the product of the probabilities. Therefore:

$S=S_{1}+S_{2}$

$f\left(\left(W_{1} \cdot W_{2}\right)=f\left(W_{1}\right)+f\left(W_{2}\right)\right.$

Equations (7) and (8) can take the entropy as a function of the probability as follows:

$S=k \ln (W)+$ constant

That $k$ is a universal constant. Equation (9) can calculate the absolute entropy. This equation also determines the general dependence between entropy and probability and als orelates the macroscopic state of the systemto its microscopic states. The probability of a physical systemdepends on the available state at a particular level of energy. In this case, increasing entropy will increase the probability of availability. Methods such as non-equilibrium statistical operator can be used to investigateirreversibility from the point of view of statistical mechanics [25,28].

\section{ENTROPY IN THE QUANTUM PERSPECTIVES OF THE STATISTICAL PHYSICS}

Using quantum concepts, the probability of availability of a particular state can be calculated. Plank [29] calculated the entropy of a physical systemas a function of its energy distortion:

$S=k[(N+P) \ln (N+P)-N \ln (N)-P \ln (P)]$

That $N$ is resonators, and $P$ is an integer. The equation of $W$ is presented for particle systems as a function of the number of particles and a weighting factor of energy levels [29]. For example, the Fermi-Dirac equation is as follows:

$W=\prod_{j} \frac{g_{j} !}{\left(g_{j}-1\right) ! N_{j} !}$

That $N_{j}$ is the number of particles with the weighting factor of energy level $g_{j}$.

As can be seen, statistical physics investigates the states of a systemby examining each particle energy state.

\section{MACROSCOPIC QUASI-STATISTICAL ENERGY STRUCTURE MODELING}

In a physical system, the instantaneous reversal of the motion of every moving particle causes the system to move backward, each particle along its old path at the same speed as before when in the same position. In physical dynamics, this simple and perfect reversibility fails, on account of forces depending on the friction of solids; imperfect fluidity of fluids; imperfect elasticity of solids; inequalities of temperature and consequent conduction of heat produced by stresses in solids and fluids; imperfect magnetic retentiveness; residual electric polarization of dielectrics; generation of heat by electric currents induced by motion; diffusion of fluids, solution of solids in fluids and other chemical changes and absorption of radiant heat and light. The dissipation of the energy investigation can be done by studying the dynamics of the particles [30-34].

When a physical system experiences a physical process, some of its energy components are activated. Activated components depend on the amount and how energy is applied to the system. In this case, when the performed processes are reversible (or the performed process is quasi-static), the least number of energy components are activated (to the diffusion of energy to be less) and the applied energy is divided between them. If the same amount of energy is applied to the system at a limited exchange rate, then, it is possible, some other components will be activated, and thus the applied energy needs to be divided between more components. 
In a quasi-static process, some components are changed independently and are dependent on how energy is applied to the system only, and some are considered dependent on the amount of other components and are therefore considered as dependent components. If the process is performed at a limited rate of energy applying, then (When the systemcan generate entropy) some secondary dependent components are also activated and take up some of the applied energy (To the diffusion energy be more). The secondary dependent components are considered to be dependent on the rate of the independent components. Therefore, each physical process can be expressed as some independent components, some primary dependent components, and some second ary dependent components. The components of the secondary dependence are dependent on how energy is applied to the system.

In the view of the independent and dependent components of energy, equation(12) is pres ented as expressing the structure of energy:

$U_{T}=\left(u_{1}+u_{2}+\cdots+u_{m}\right)+\left[g_{1}+\cdots+g_{k}\right]+\left[h_{1}+\cdots+h_{n}\right]+U_{T_{0}}$

Where:

$g_{j}=g_{j}\left(u_{1}, u_{2}, \ldots, u_{m}\right)$

$h_{p}=h_{p}\left(\dot{u}_{1}, \ldots, \dot{u}_{m}\right)$

The functions $g_{j}$ represent the value-dependent components of the independent components, while the functions $h_{p}$ show the components dependent on the rate of the independent components. Als o $U_{T_{0}}$ represents the sumof all the components that did not participate in the performed process. These components are macroscopic and are the result of a specific type of energy in part or all of the system's particles. Equation(12) is formed according to the performed process and also how the energy is applied to the system and makes it possible to consider the effects of how the energy exchange can be considered in the energy structure of the system.

Equation (12) is in the same line with the second law. Based on the differentformulations of the second law, some of the av ailable work must be converted to thermal energy and we know that the thermalenergy depend s on the kinematic of substructures [35] while other types of energies depend on the place as well as displacement of sub-structures.

\section{IRREVERSIBILITY ANALYSIS}

To investigate the irreversibility, it is necessary to assume that some energy is applied to the system in different paths [25]. Then to examine the changes in the energy components of the system, initially, it is as sumed that theenergy applied to the system is such that it activates an independent component, and then the results will be generalized to processes with the desired number of the independent components.

Therefore, it should be as sumed that the component $u_{i}$ is the only independent component activated in the performed process, as can be seen in figure 1.

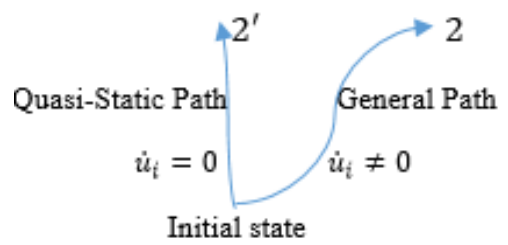

Figure 1. Different paths for the performed process

In this case, the energy structure of the systemcan be written as follows:

$\delta U_{T}=u_{i}+\sum_{j=1}^{k} g_{j}\left(u_{i}\right)+\sum_{p=1}^{n} h_{p}\left(u_{i}\right)+U_{T_{0}}$

If the energy $\delta U_{T}$ is applied to the system in a quasi-static path $\left(\dot{u}_{i}=0\right)$ and as a result the independent component of energy changes to the amount of $\delta u^{\prime}{ }_{i}$, then it can be written:

$\delta U_{T}=\delta u_{i}^{\prime}+\sum_{j=1}^{k}\left(\frac{\partial g_{j}}{\partial u_{i}} \delta u_{i}^{\prime}\right)$

And if the same amount of energy is applied to the systemin general $\left(\dot{u}_{i} \neq 0\right)$, it can be written:

$\delta U_{T}=\delta u_{i}+\sum_{j=1}^{k}\left(\frac{\partial g_{j}}{\partial u_{i}} \delta u_{i}\right)+\sum_{p=1}^{n}\left(\frac{\partial h_{p}}{\partial \dot{u}_{j}} \delta \dot{u}_{i}\right)$ 
Therefore, it is neces sary that:

$$
\left(1+\sum_{j=1}^{k}\left(\frac{\partial g_{j}}{\partial u_{i}}\right)\right)\left(\delta u_{i}-\delta u_{i}^{\prime}\right)=-\left(\sum_{p=1}^{n}\left(\frac{\partial h_{p}}{\partial \dot{u}_{i}}\right)\right) \delta \dot{u}_{i}
$$

Given that the amount of energy applied to the systemis given at a limited rate $\left(\dot{u}_{i} \neq 0\right)$, this energy is divided into more components, and if only changes due to this process will be considered no otherinner possible processes, then $\delta u^{\prime}{ }_{i} \geq \delta u_{i}$ for the conditions that $\delta U_{T}>0$ and $\delta u_{i}^{\prime} \leq \delta u_{i}$ for the conditions that $\delta U_{T}<0$. Given that the primary dependent components, $g_{j}$, will always be activated in all paths (since they are dependent on to the independent components that, for a particular process, will be activated always), these components must be satisfied $\sum_{j=1}^{k}\left(\frac{\partial g_{j}}{\partial u_{i}}\right) \geq 0$ for all possible values for $u_{i}$. Therefore, according to the equation (18), if $\delta U_{T}>0$ in this case $\left(\sum_{p=1}^{n}\left(\frac{\partial h_{p}}{\partial \dot{u}_{i}}\right)\right) \delta \dot{u}_{i}>0$ and if $\delta U_{T}<0$, so $\left(\sum_{p=1}^{n}\left(\frac{\partial h_{p}}{\partial \dot{u}_{i}}\right)\right) \delta \dot{u}_{i}<0$. Also, if the process is quasi-s tatic $\left(\dot{u}_{i}=0\right)$, in this case $\left(\sum_{p=1}^{n}\left(\frac{\partial h_{p}}{\partial \dot{u}_{i}}\right)\right) \delta \dot{u}_{i}=0$.

Therefore, considering $\delta H_{i}=\left(\sum_{p=1}^{n}\left(\frac{\partial h_{p}}{\partial \dot{u}_{i}}\right)\right) \delta \dot{u}_{i}$, the quantities $H_{i}$ can be a good basis for examining irreversibility (or entropy generation) to the component $u_{i}$. In addition to the internal structure of the system, as well as its current state, this quantity als o depends on how energy is applied to the system.

Also, the value $\left(\delta u_{i}-\delta u_{i}^{\prime}\right)$ can be considered as the deviation fromthe quasi-static path to the general, as can be seen in figure 2 .

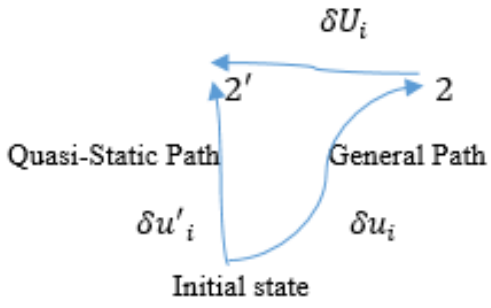

Figure 2. Deviation fromthe quasi-s tatic path to the general

Therefore, by the variable changes $\delta U_{i}=\left(\delta u_{i}-\delta u_{i}^{\prime}\right)$, the variation $\delta H_{i}$ can be rewritten as follows:

$\delta H_{i}=\left(\sum_{p=1}^{n}\left(\frac{\partial h_{p}}{\partial \dot{U}_{i}}\right)\right) \delta \dot{U}_{i}$

Which gives $\delta H_{i}$ according to how energy is applied to the systemas well as its inner physical structure.

Also, the stated concepts can be generalized to the other independent components. So, the following equation can be considered:

$\delta \Psi=\sum_{i=1}^{m} \delta H_{i}$

In this case, if $\delta U_{T}>0$ then $\Psi \delta \geq 0$ and if $\delta U_{T}<0$ then $\Psi \delta \leq 0$ and also in the quasi-static path, $\Psi \delta=0$. So by defining:

$\varphi=\frac{\delta \Psi}{\delta U_{T}}=\sum_{i=1}^{m} \frac{\delta H_{i}}{\delta U_{T}}=\sum_{i=1}^{m} \varphi_{i}$

In this case, always $\varphi \geq 0$ (as well as $\varphi_{i} \geq 0$ ). Therefore, $\varphi$ can be used as a criterion for calculating irreversibility (or generated entropy). And also $\varphi_{i}$ will be an irreversible criterion for the energy component $u_{i}$. This quantity depends on how energy is applied to the systemas well as its amount and inner physical structure of the system.

Equation (21) shows the irreversibility structure and its components. Due to this equation, entropy is generated because of the independent energy components activated in performed physical processes. These components will not be activated in a quasi-s tatic path (zero rates of applying energy) and will be activated when the rate of applying energy is non-zero (general paths). Due to the kinematic theory of dis sipated energy, these results are in the same line with the second law of thermodynamics [30-32].

Equation (21) has been extracted u sing the macro scopic energy components and don't need to investigate all sub-structures. Therefore, if equation (21) to be used to investigate irreversibility in configurationally systems, macroscopic energy components are enough for relevant analy sis. 


\section{CONCLUSIONS}

Entropy production is one of the most important concepts introduced by the second law of thermodynamics that can be occurred during a physical process. From the perspective of classical thermodynamics, entropy can be generated when the processes are performed at a non-zero rate and means that some of the applied energy los ses to the thermal energy.

From the statis tical physics point of view, the Boltzmann equation investigates microstates of the systemand entropy can be calculated. The Boltzmann equation also can be used for physical systems that particles have no many degrees of freedom, as configurationally entropy. From the perspective of the statistical physics (Boltzmann equation), configurationally entropy can be calculated using the study of the microstates of the system. When a physical process is performed, identifying entropy production can be us ed to investigating the irreversibility, but from the perspective of the Boltzmann equation, to study the entropy production, both micros tates and macrostates mus t be studied. Therefore, more of course to study a configurationally system, a large volume of calculations are required.

In this study, a new macroscopic quasi-s tatistical approach is presented to investigate the irreversibility. Using the study of the macro energy components of the system, the irreversibility structure can be identified. Therefore using this, a new macros copic definition of the generated entropy (Eq. 21) is extracted using energy structure equation analy sis in different paths as well as macros copic dependent and independent energy component. This equation can be used to investig ate the entropy production without the need to study all sub-structures. Therefore, it can be used for studying configurationally systems easily.

To inves tigate why and what entropy can be generated during physical processes, the energy structure of the system can be studied. Equation (12) is pres ented as the energy structure equation of the system and is determined according to the macroscopic components of the energy and is formed according to the performed process and also how the energy is applied to the system as well as the amount of the applied energy. In fact, it was assumed that some energy would be given to the systemonce in a quasi-s tatic path and again in general. By this, using the energy structure equation, it can be detected that the condition of the applied energy how can changes the amount of the internal variation of the energy components. As a result, equation (21), by defining a quantity always greater than or equal to zero $\varphi$, is obtained as a criterion for the structure of irreversibility (or generated entropy) as well as its components $\left(\varphi_{i}\right)$.

Also, the extracted equations result that entropy is generated because of the existence of the dependent energy components in the energy structure of the systemand this generated entropy depends on the variation of these components as well as the amount of the applied energy to the system and its conditions. These results are in the same line with the different formulations of the second law of thermodynamics.

\section{REFERENCES}

[1] Sheehan, D. P. "The second law of thermodynamics: Foundations and status." Foundations of Physics 37.12 (2007): 1653-1658.

[2] Erlichs on, Herman. "Sadi Carnot,Founder of the Second Law of Thermodynamics'." European journal ofphysics 20.3 (1999): 183.

[3] Kostic, Milivoje M. "Revisiting the second law of energy degradation and entropy generation: From Sadi Carnot's ingenious reasoning to Holistic generalization." AIP Conference Proceedings. Vol. 1411. No. 1. American Institute of Physics, 2011.

[4] Carnot, Sadi, RudolfClausius, and William Thoms on Baron Kelvin. The Second Law ofThermodynamics. American Book Company, 1899.

「5] Goldstein, Sheldon. "Boltzmann's approach to statistical mechanics." Chance in physics. Springer, Berlin, Heidelberg, 2001. 39-54.

[6] Wallace, David. "Probability and irreversibility in modern statistical mechanics: Classical and quantum." Quantum Foundations of Statistical Mechanics (Oxford University Press, forthcoming ) (2016).

[7] Burbury, S. H. "LVII. The second law of Thermodynamics." The London, Edinburgh, and Dublin Philosophical Magazine and JournalofScience 37.229 (1894): 574-578.

[8] Carnot, Sadi, RudolfClausius, and William Thoms on Baron Kelvin. The Second Law ofThermodynamics. American Book Company, 1899.

[91 Lieb, Elliott H., and Jakob Yngvason. "The physics and mathematics of the second law of thermodynamics." Physics Reports 310.1 (1999): 1-96.

「101 Buchdahl, Hans Adolph. "On the unrestricted theorem of Carathéodory and its application in the treatment of the second law of thermodynamics." American Journal of Physics 17.4(1949): 212-218.

[11] Eis ens chitz, R. "The principle of Carathéodory." Science Progress (1933-) 43.170 (1955): 246-260.

[12] Buchdahl, H. A. "On the principle of Carathéodory." American Journal of Physics 17.1 (1949): 41-43.

[13] Redlich, Otto. "Fundamental thermodynamics since Carathéodory." Reviews of Modern Physics 40.3 (1968): 556.

[14] Greven, Andreas, Gerhard Keller, and Gerald Warnecke, eds. Entropy. Vol. 47. Princeton University Press, 2014. 
[15] Bailey, Kenneth D. "Entropy systems theory." Systems Science and Cybernetics, Eolss Publishers, Oxford, UK (2009): 152-169.

[16] Daub, Edward E. "Entropy and dissipation." Historicalstudies in the physical sciences 2(1970): 321-354.

[17] Wehrl, Alfred. "General properties of entropy." Reviews of Modern Physics 50.2 (1978): 221.

[18] Klein, Joseph Frederic. Physical significance of entropy or of the second law. D. Van Nostrand Company, 1910.

[19] Garver, M. M. "On the Laws of Energy and the Physical Significance of Entropy." The Journal of Physical Chemistry 15.7 (2002): 613-638.

[20] Chakrabarti, C. G., and Indranil Chakrabarty. "Boltzmann entropy: probability and information." arXiv preprint arXiv:0705.2850(2007).

[21] Bekenstein, Jacob D. "Generalized second law of thermodynamics in black-hole physics." Physical Review D 9.12 (1974): 3292.

[22] Karami, K., and A. Abdolmaleki. "Generalized second law of thermodynamics in $\mathrm{f}(\mathrm{T})$ gravity." Journal of Cosmology and Astroparticle Physics 2012.04 (2012): 007.

[23] Zhou, Jia, et al. "The generalized second law of thermodynamics in the accelerating universe." Physics Letters $B$ 652.2-3 (2007): 86-91.

[24] Bamba, Kazuharu, et al. "Generalized second law of thermodynamics in $f$ (T) gravity with entropy corrections." Astrophysics and Space Science 344.1 (2013): 259-267.

[25] Luzzi, Roberto, Áurea R. Vasconcellos, and J. Galvão Ramos. "On the statistical foundations of irreversible thermodynamics." Fortschritte der Physik: Progress of Physics 47.4(1999): 401-432.

[26] Onsager, Reciprocal relations in irreversible processes I. Phys. Rev. 37, 405 (1931); ibid. II 38, 2235 (1931).

[27] Coveney, Peter V. "The second law of thermodynamics: entropy, irreversibility and dynamics." Nature 333.6172 (1988): 409-415.

[28] Lucia, Umberto. "Statis tical approach of the irreversible entropy variation." Physica A: Statistical Mechanics and its Applications 387.14 (2008): 3454-3460.

[29] Cohen, E. G. D. "Boltzmann and Einstein: Statistics and dynamics—an unsolved problem." Pramana 64.5 (2005): 635-643.

[30] Thomson, William. "9. The kinetic theory of the dissipation of energy." Proceedings of the Royal Society of Edinburgh 8(1875): 325-334.

[31] Burbury, S. H. "LII. A theorem on the dissipation of energy." The London, Edinburgh, and Dublin Philosophical Magazine and JournalofScience 13.83 (1882): 417-419.

[32] Rayleigh, Lord. "On the dissipation of energy." Van Nostrand's Eclectic Engineering Magazine (1869-1879) 12.78 (1875): 519.

「33] Thoms on, William. "2. On a Univers al Tendency in Natu reto the Dis sipation of Mechanical Energy." Proceedings of the Royal Society of Edinburgh 3 (1857): 139-142.

[34] Esmaeilpour, Morteza, and Maziar Gholami Korzani. "Enhancement of immis cible two-phase dis placement flow by introducing nanoparticles and employing electro-and magneto-hydrodynamics." Journal of Petroleum Science and Engineering 196: 108044.

[35] Eu, Byung C. "Kinetic theory and irreversible thermodynamics." NASA STI/Recon Technical Report A 93(1992). 